\title{
The Relationship between Bleeding and Original Contrast Extravasation Judged by Dual Energy CT after Acute Thrombectomy
}

Tomohiro lida, ${ }^{1,2}$ Keita Yamauchi, ${ }^{1}$ Shunsuke Takenaka, ${ }^{3}$ and Hideki Sakai ${ }^{1}$

Objective: There are many cases in which computed tomography (CT) after acute thrombectomy demonstrates high-density areas, but it may be difficult to judge whether this is hemorrhage or contrast extravasation. Dual energy CT (DECT) is an imaging method that enables discrimination of substances by acquiring $\mathrm{X}$-ray image data of two different energies.

Methods: We performed DECT to distinguish hemorrhage from contrast extravasation in cases with high-density areas on CT after acute thrombectomy at our hospital, and we compared with T2*-weighted image on the following day.

Results: Six patients comprising 22 areas had high-density areas on CT after acute thrombectomy. In all, 20 of the 22 high-density areas were determined to be contrast extravasation by DECT, and no cases of subsequent symptomatic cerebral hemorrhage were observed. However, 11 areas with new microbleeds were confirmed in the 20 extravasation areas on MRI-T2* images the day after thrombectomy.

Conclusion: This examination suggested that the contrast extravasation and its concentration are involved in the presence of low-intensity areas on $\mathrm{T}^{*}$.

Keywords $>$ dual energy CT, thrombectomy, micro bleeds, contrast extravasation

\section{Introduction}

In many patients, computed tomography (CT) after acutephase thrombectomy reveals high-density areas. We therefore examined which of two factors, hemorrhage and contrast medium extravasation, is associated with the high-density areas based on the site/shape of this area, presence of shift, and $\mathrm{CT}$ value, but it was difficult to evaluate this in some

${ }^{1}$ Department of Neurosurgery, National Hospital Organization Toyohashi Medical Center, Toyohashi, Aichi, Japan

${ }^{2}$ Department of Neurosurgery, Gifu Prefectural General Medical Center, Gifu, Gifu, Japan

${ }^{3}$ Department of Neurosurgery, Japan Organization of Occupational Health and Safety Hamamatsu Rosai Hospital, Hamamatsu, Shizuoka, Japan

Received: October 31, 2019; Accepted: March 23, 2020 Corresponding author: Tomohiro Iida. Department of Neurosurgery, Gifu Prefectural General Medical Center, 4-6-1, Noisshiki, Gifu, Gifu 500-8717, Japan

Email: bass_nathan_east@yahoo.co.jp

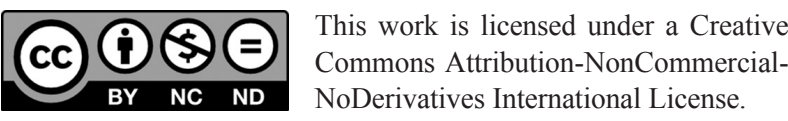

(C)2020 The Japanese Society for Neuroendovascular Therapy patients. As the presence of hemorrhage influences postoperative management, the necessity of developing an examination procedure that facilitates the differentiation of hemorrhage from contrast medium extravasation has been emphasized. Dual energy CT (DECT) is an imaging method that facilitates the discrimination of substances through the acquisition of X-ray image data in two different energy areas. It may also be useful for differentiating hemorrhage from contrast medium extravasation. A previous study reported its usefulness. ${ }^{1)}$ In this study, we performed DECT to differentiate hemorrhage from contrast medium extravasation in patients in whom CT after acutephase thrombectomy demonstrated high-density areas, and compared the results with $\mathrm{T} 2 *$-weighted images the following day to investigate the association of contrast medium extravasation with hemorrhage.

\section{Subjects and Methods}

Of nine patients who underwent acute-phase thrombectomy at our hospital between November 2018 and June 2019, we simultaneously performed plain CT and DECT for six in whom CT after treatment demonstrated 


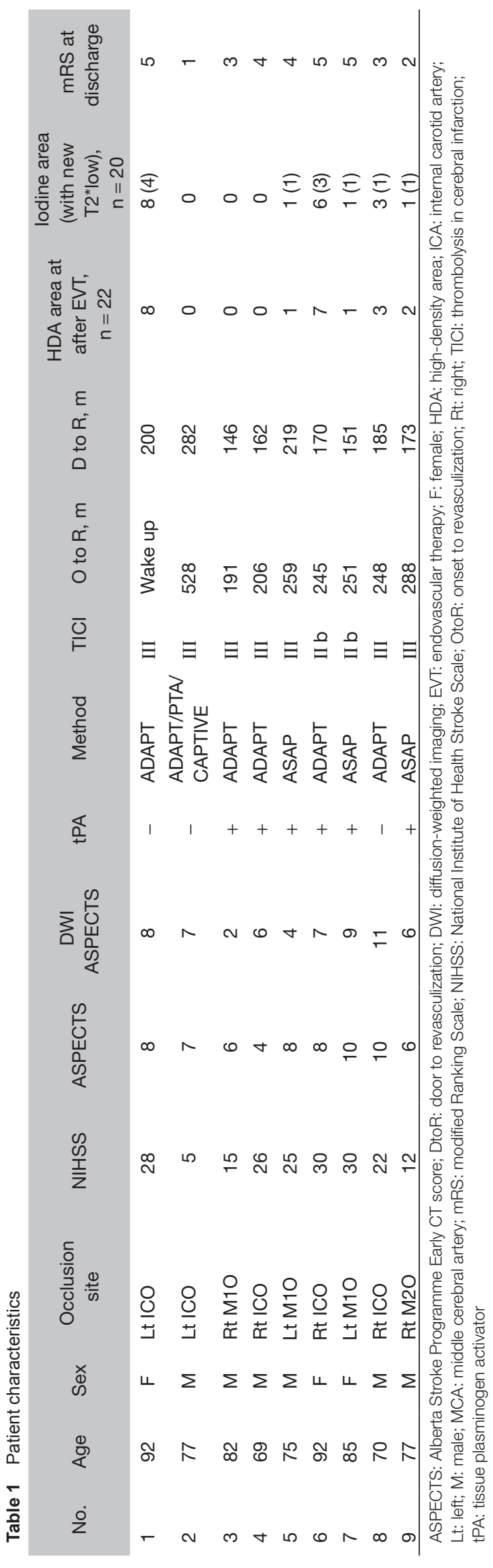

high-density areas, and conducted magnetic resonance imaging (MRI), including T2*-weighted imaging, the following day to examine its association with DECT. For DECT, an Aquillion PRIME (Toshiba, Japan) was used. The following imaging conditions were adopted: dual energy volume scan (two-rotation system); tubal voltage, $80 / 135 \mathrm{kVp}$; tubal current, 600/200 mA; collecting detector, $0.5 \mathrm{~mm} \times 80$ rows; tube rotation speed, 1 second; rearrangement function, $\mathrm{FC13}$; and rearrangement slice thickness, $5 \mathrm{~mm}$. The authors were responsible for image assessment of the presence of a high-density area on CT after treatment. We confirmed the area of contrast medium on an iodine map obtained using DECT and measured the $\mathrm{CT}$ values at several points per area. The greatest value was regarded as the maximum in the area. In our hospital, a direct aspiration first pass technique (ADAPT) with a Penumbra system (Penumbra Alameda, CA, USA) was selected as the first-choice procedure for thrombectomy. When a thrombus was impossible to aspirate, treatment was performed using different stent retrievers based on the conditions in individual patients.

\section{Results}

In six (four males and two females) of the nine patients, CT after acute-phase thrombectomy demonstrated highdensity areas, with a mean age of 79.9 years (Table 1). Among them, the internal carotid artery and the middle cerebral artery were affected in three patients each (M1: two patients, M2: one patient). In four patients, thrombectomy had been performed after the administration of recombinant tissue plasminogen activator (rt-PA). In the other two patients for whom rt-PA was not indicated, thrombectomy alone had been conducted. For all patients, there was diffusion-weighted imaging (DWI)-fluid-attenuated inversion recovery (FLAIR) mismatch, suggesting early after onset, or DWI-clinical mismatch, suggesting the penumbra region. Concerning the results of treatment, TICI3 recanalization was achieved in four patients and TICI $2 \mathrm{~b}$ recanalization was achieved in two patients. In these six patients, CT immediately after treatment revealed high-density areas at 22 points. At 20 of these points, contrast medium was detected on DECT (20/22 points, 90.9\%). All areas where contrast medium was detected were within ischemic areas on $\mathrm{CT}$ or DWI on arrival or the latter the following day. Case 1 is presented in Fig. 1. In Case 1, CT immediately after treatment demonstrated eight high-density areas and contrast medium was detected in all areas on DECT. In 

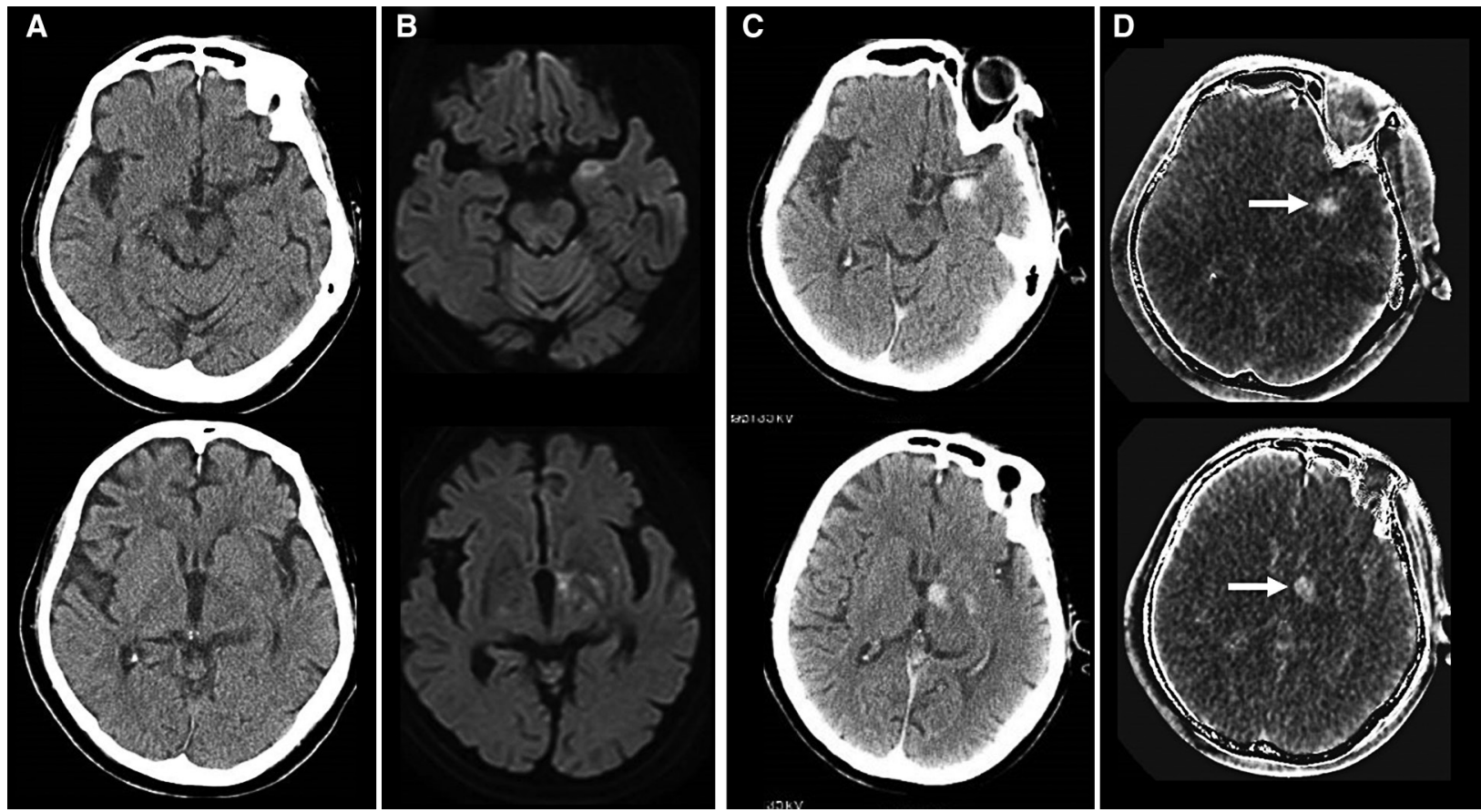

Fig. 1 A 92-year-old female (Case 1). CT (A)/diffusion-weighted imaging (B) before thrombectomy. A high-density area on CT (C) after thrombectomy was confirmed to be contrast medium extravasation (arrow) on an iodine map (D). CT: computed tomography
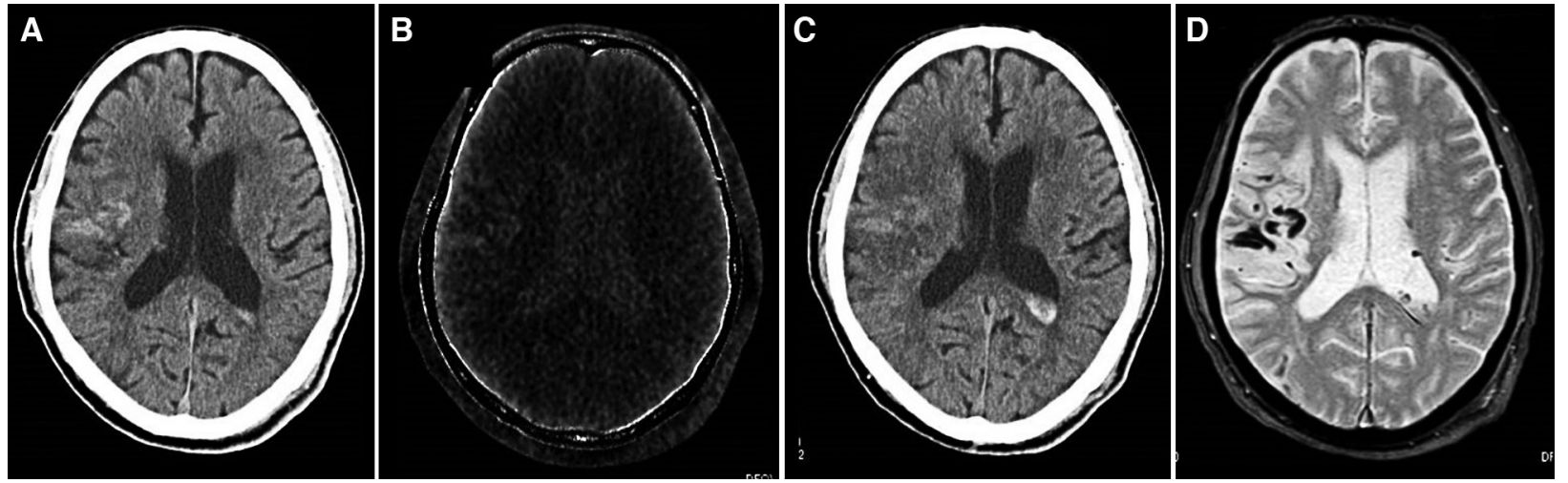

Fig. 2 A 77-year-old male (Case 9). CT (A) and iodine map (B). CT the following day showed a high-density area (C) at the site of contrast medium detection. T2*-weighted imaging (D) revealed hemorrhage, suggesting the concomitant existence of contrast medium and hemorrhage. CT: computed tomography

two of the rt-PA-treated patients, cerebellar or intraventricular hemorrhage was observed regardless of ischemic areas immediately after treatment. However, CT during the course did not reveal cerebral hemorrhage in any other patient. At 19 of the 20 points where contrast medium was detected, it was washed out on CT the following day, excluding one patient. One point where a light highdensity area remained was confirmed in Case 9 , and T2* weighted imaging the following day revealed hemorrhage at the same site, suggesting the simultaneous presence of contrast medium and hemorrhage (Fig. 2). As presented in Table 2, six patients in whom CT after surgery demonstrated high-density areas were compared with three without such areas. There were no marked differences in the age (mean: $81.8 \pm 8.4$ vs. $76.0 \pm 5.4$ years, respectively), site of occlusion (IC: three patients, M1: 2, M2: 1 vs. IC: 2, M1: 1, respectively), tPA administration (4/6 patients vs. $2 / 3$ patients, respectively), or door-to-recanalization time (mean: $183.0 \pm 21.9$ vs. 196.760.7 minutes, respectively), but the mean national institute of health stroke scale (NIHSS) score (24.5 \pm 6.3 vs. $15.3 \pm 8.6$, respectively) was slightly higher in the six patients with high-density areas. The Alberta stroke programme early CT score (ASPECTS)/DWI-ASPECTS 

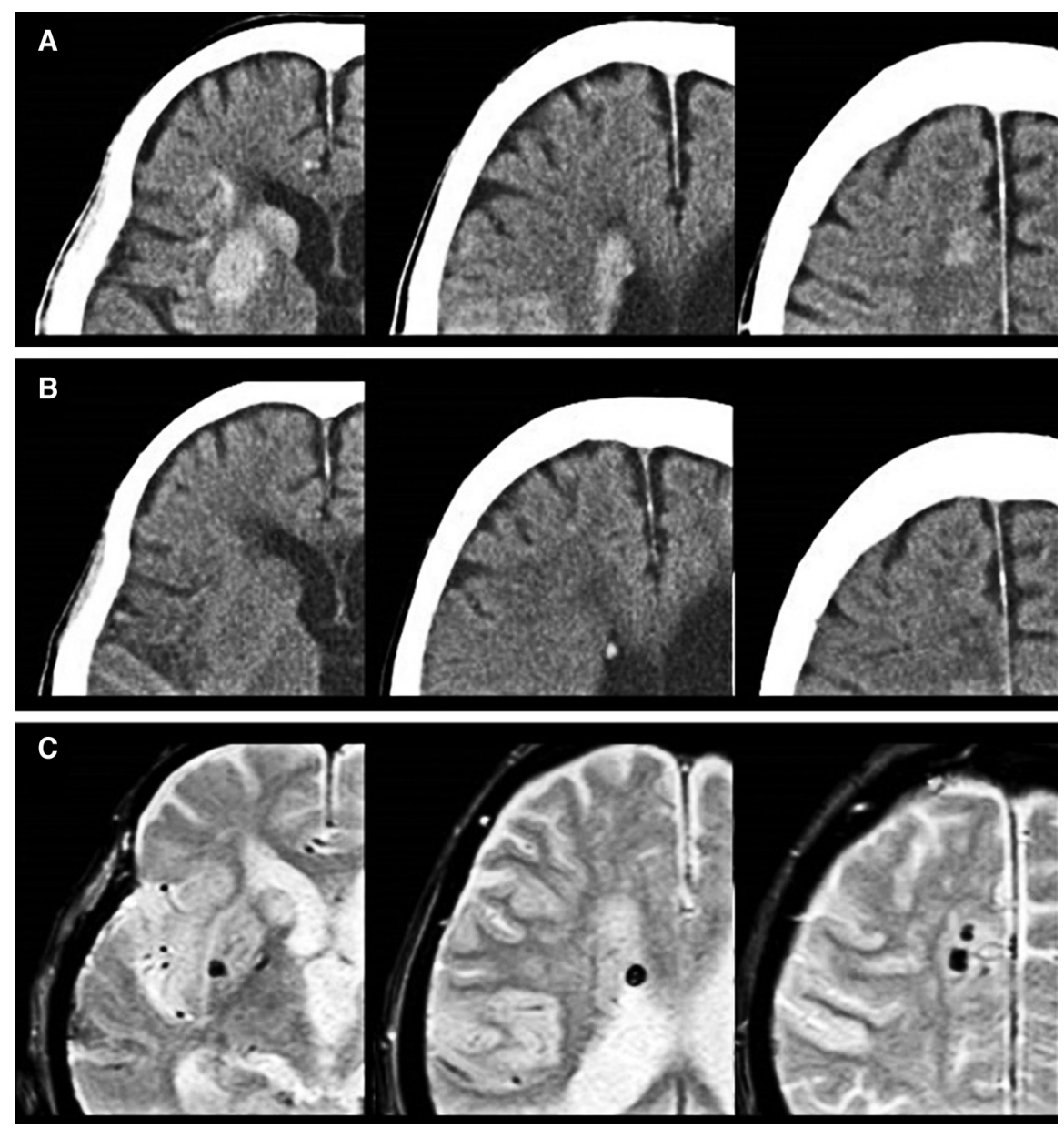

Fig. 3 A 92-year-old female (Case 6). CT (B) the following day confirmed washout in a high-density area on CT (A). Furthermore, T2*-weighted MRI (C) revealed fresh microhemorrhage in a portion of the same area. CT: computed tomography; MRI: magnetic resonance imaging

Table 2 Case comparison regarding the presence of high-density areas

\begin{tabular}{lcc} 
& $\begin{array}{c}\text { HDA }+ \\
(n=6)\end{array}$ & $\begin{array}{c}\text { HDA- } \\
(n=3)\end{array}$ \\
Age, $y$ & $81.8 \pm 8.4$ & $76.0 \pm 5.4$ \\
$\begin{array}{l}\text { Occlusion site, } n \\
\text { ICA }\end{array}$ & & \\
M1 & 3 & 2 \\
M2 & 2 & 1 \\
NIHSS score & 1 & \\
ASPECTS, score & $24.5 \pm 6.3$ & $15.3 \pm 8.6$ \\
DWI-ASPECTS, score & $8.3 \pm 1.4$ & $5.7 \pm 1.2$ \\
D to R, m & $7.5 \pm 2.2$ & $5.0 \pm 2.2$ \\
\hline ASPECTS: Alberta Stroke Programme Early CT score; DtoR: door to \\
revasculization; DWl: diffusion-weighted imaging; HDA: high-density \\
area; ICA: internal carotid artery; M1 and M2: middle cerebral artery
\end{tabular}

$(8.3 \pm 1.4 / 7.5 \pm 2.2$ vs. $5.7 \pm 1.2 / 5.0 \pm 2.2$, respectively $)$ was higher in the six patients.

Case 6 is presented in Fig. 3. In Case 6, contrast medium was detected in all six high-density areas (excluding cerebellar hemorrhage) on CT immediately after treatment, and it was washed out on CT the following day. However, at three of the six points, new low-intensity areas were confirmed on $\mathrm{T} 2 *$-weighted imaging. Of 20 areas (six patients) where contrast medium was detected on DECT, a new T2* low-intensity area, which was not observed before treatment, was detected in 11 (55\%). We compared the maximum CT value on DECT between the 11 points and 9 non-low-intensity areas. The mean maximum CT values in the former and latter were 55.0 \pm 15.7 and $47.3 \pm 13.8 \mathrm{HU}$, respectively, with no significant difference. However, the value may be slightly higher in the former.

\section{Discussion}

An entity of DECT was proposed in the 1970s. DECT systems became commercially available at the beginning of the 2000s. As changes in the CT value related to X-ray 
energy changes are specific to substances, DECT is a procedure by which imaging at different voltages (high and low voltages) facilitates the differentiation of substances. Currently, many devices are available for such imaging, and are useful for differentiating hemorrhage from contrast medium extravasation. In addition, these devices have been clinically applied to remove beam hardening artifacts or bone/calcification. ${ }^{2)}$

In the six patients treated at our hospital, CT after surgery demonstrated 22 high-density areas. Of these, two were associated with cerebellar or intraventricular hemorrhage, which was not related to infarction. However, the other 20 areas were associated with contrast medium extravasation on DECT. CT the following day confirmed washout in the areas, excluding one (CT was not performed for one patient; eight points). Thus, conditions were able to be accurately evaluated in most sites (11/12 points; $91.7 \%)$, as described in previously. ${ }^{1)}$

We compared six patients in whom CT after surgery revealed high-density areas with three without such areas. There were no marked differences in the age, site of occlusion, t-PA administration, or interval from arrival until recanalization. In the six patients, the NIHSS score was slightly higher and the ASPECTS/DWI-ASPECTS was higher. However, the number of subjects was small and there were no significant differences.

Furthermore, delayed cerebral hemorrhage after thrombectomy is a frequent complication, influencing the prognosis. Therefore, many studies reported the prediction of delayed cerebral hemorrhage. Takei et al. examined the ASPECTS and NIHSS score before thrombectomy, collateral score, and edema in infracted lesions (net water uptake) as risk factors. ${ }^{3)}$ Nakano et al. ${ }^{4)}$ reported that the incidence of hemorrhage with subsequent neurological deficits in patients with high-density areas on CT after thrombectomy was significantly higher than in those without such areas (29.7 vs. 0\%, respectively). In addition, Bonatti et al. suggested that the risk of delayed cerebral hemorrhage is significantly higher in patients with a maximum contrast medium concentration of $\geq 1.35 \mathrm{mgI} / \mathrm{mL}$ among those with high-density areas (sensitivity: 100\%, specificity: $67.6 \%){ }^{5}$ )

Pathophysiologically, vascular-wall rupture and an ischemia-related increase in blood-brain barrier (BBB) permeability may be involved in the pathogenesis of postthrombectomy hemorrhage. As $\leq 400$-Da substances alone may permeate the $\mathrm{BBB}$, a previous study reported that contrast medium leakage or hemorrhage can be evaluated as different phases of BBB disturbance. $\left.{ }^{6}\right)$ In our patients, T2*weighted imaging confirmed the new development of microhemorrhage in $\geq 50 \%$ (11/20 points) of the areas where contrast medium was detected, suggesting that the detection of contrast medium on DECT is a risk factor for hemorrhage. Among the areas where contrast medium was detected, the CT value on DECT in low-intensity areas on T2*-weighted MRI the following day was slightly higher than that in non-low-intensity areas $(55.0 \pm 15.7$ vs. $47.3 \pm$ 13.8 HU, respectively), although there was no significant difference. This suggests that the concentration of contrast medium played a role in microhemorrhage. Briefly, a high concentration of contrast medium may reflect a more advanced phase of BBB disturbance.

The detection of contrast medium is influenced by the frequency of intraoperative contrast-enhanced imaging, infusion pressure, and presence of imaging through a microcatheter; therefore, there may be procedure-related changes. In addition, whether DECT should be performed after thrombectomy is evaluated based on the presence of high-density areas, a subjective factor; the possibility that high-density areas may be overlooked cannot be excluded. In addition, this study involved a small number of patients, and this issue should be further investigated in a larger number of patients in the future.

\section{Conclusion}

Contrast medium was able to be detected on DECT after thrombectomy. We compared DECT with T2*-weighted MRI, and suggest that the detection of contrast medium using DECT is a risk factor for post-treatment hemorrhage. Therefore, DECT after thrombectomy may provide information useful for postoperative blood pressure control or examining the timing of starting antithrombotic therapy.

\section{Disclosure Statement}

We declare no conflict of interest.

\section{References}

1) Phan CM, Yoo AJ, Hirsch JA, et al: Differentiation of hemorrhage from iodinated contrast in different intracranial compartments using dual-energy head CT. AJNR Am J Neuroradiol 2012; 33: 1088-1094.

2) Naruto $N$, Itoh $T$, Noguchi $K$ : Dual energy computed tomography for the head. Jpn J Radiol 2018; 36: 69-80. 
3) Takei J, Irie $K$, Tanaka $T$, et al: Evaluation of the CT high-density area after endovascular treatment for acute ischemic stroke. JNET J Neuroendovasc Ther 2017; 11: 227-234

4) Nakano S, Iseda T, Kawano H, et al: Parenchymal hyperdensity on computed tomography after intra-arterial reperfusion therapy for acute middle cerebral artery occlusion incidence and clinical significance. Stroke 2001; 32: 2042-2048.
5) Bonatti M, Lombardo F, Zamboni GA, et al: Iodine extravasation quantification on dual-energy CT of the brain performed after mechanical thrombectomy for acute ischemic stroke can predict hemorrhagic complications. AJNR Am J Neuroradiol 2018; 39: 441-447.

6) Renú A, Amaro S, Laredo C, et al: Relevance of bloodbrain barrier disruption after endovascular treatment of ischemic stroke: dual-energy computed tomographic study. Stroke 2015; 46: 673-679. 